\title{
A degree sum condition on the order, the connectivity and the independence number for hamiltonicity
}

\author{
Shuya Chiba* \\ Applied Mathematics, Faculty of Advanced Science and Technology \\ Kumamoto University \\ 2-39-1 Kurokami, Kumamoto 860-8555, Japan \\ schiba@kumamoto-u.ac.jp \\ Michitaka Furuya ${ }^{\dagger}$ \\ College of Liberal Arts and Sciences \\ Kitasato University \\ 1-15-1 Kitasato, Minami-ku, Sagamihara, Kanagawa 252-0373, Japan \\ michitaka.furuya@gmail.com \\ Kenta Ozeki ${ }^{\ddagger}$ \\ Faculty of Environment and Information Sciences \\ Yokohama National University \\ 79-7 Tokiwadai, Hodogaya-ku, Yokohama 240-8501, Japan \\ ozeki-kenta-xr@ynu.ac.jp \\ Masao Tsugaki \\ Department of Applied Mathematics \\ Tokyo University of Science \\ 1-3 Kagurazaka, Shinjuku-ku, Tokyo 162-8601, Japan \\ tsugaki@hotmail.com \\ Tomoki Yamashita ${ }^{\S}$ \\ Department of Science \\ Kindai University \\ 3-4-1 Kowakae, Higashi-Osaka, Osaka 577-8502, Japan \\ yamashita@math.kindai.ac.jp
}

Submitted: Aug 10, 2015; Accepted: Dec 2, 2019; Published: Dec 20, 2019

(c) The authors. Released under the CC BY-ND license (International 4.0). 


\begin{abstract}
In [Graphs Combin. 24 (2008) 469-483], the third author and the fifth author conjectured that if $G$ is a $k$-connected graph such that $\sigma_{k+1}(G) \geqslant|V(G)|+\kappa(G)+$ $(k-2)(\alpha(G)-1)$, then $G$ contains a Hamilton cycle, where $\sigma_{k+1}(G), \kappa(G)$ and $\alpha(G)$ are the minimum degree sum of $k+1$ independent vertices, the connectivity and the independence number of $G$, respectively. In this paper, we settle this conjecture. The degree sum condition is best possible.
\end{abstract}

Mathematics Subject Classifications: 05C38, 05C45

\title{
1 Introduction
}

\subsection{Degree sum condition for graphs with high connectivity to be hamilto- nian}

In this paper, we consider only finite undirected graphs without loops or multiple edges. For standard graph-theoretic terminology not explained, we refer the reader to [5].

A Hamilton cycle of a graph is a cycle containing all the vertices of the graph. A graph having a Hamilton cycle is called a hamiltonian graph. The hamiltonian problem has long been fundamental in graph theory. Many researchers have investigated sufficient conditions for a graph to be hamiltonian. In this paper, we deal with a degree-sum-type condition, which is one of the main stream of this study.

We introduce four invariants, including degree sum, which play important roles for the existence of a Hamilton cycle. Let $G$ be a graph. The number of vertices of $G$ is called its order, denoted by $n(G)$. A set $X$ of vertices in $G$ is called an independent set in $G$ if no two vertices of $X$ are adjacent in $G$. The independence number of $G$ is the maximum cardinality of an independent set in $G$, denoted by $\alpha(G)$. For two distinct vertices $x, y \in V(G)$, the local connectivity $\kappa_{G}(x, y)$ is defined to be the maximum number of internally-disjoint paths connecting $x$ and $y$ in $G$. A graph $G$ is $k$-connected if $\kappa_{G}(x, y) \geqslant k$ for any two distinct vertices $x, y \in V(G)$. The connectivity $\kappa(G)$ of $G$ is the maximum value of $k$ for which $G$ is $k$-connected. We denote by $N_{G}(x)$ and $d_{G}(x)$ the neighborhood and the degree of a vertex $x$ in $G$, respectively. If $\alpha(G) \geqslant k$, let

$$
\sigma_{k}(G):=\min \left\{\sum_{x \in X} d_{G}(x): X \text { is an independent set in } G \text { with }|X|=k\right\}
$$

otherwise let $\sigma_{k}(G):=+\infty$. If the graph $G$ is clear from the context, we simply write $n$, $\alpha, \kappa$ and $\sigma_{k}$ instead of $n(G), \alpha(G), \kappa(G)$ and $\sigma_{k}(G)$, respectively.

\footnotetext{
*Supported by JSPS KAKENHI Grant Number 17K05347.

†Supported by JSPS KAKENHI Grant Number 18 K13449.

${ }^{\ddagger}$ Supported by JST ERATO Kawarabayashi Large Graph Project, Grant Number JPMJER1201, Japan, and JSPS KAKENHI Grant Number 18K03391.

${ }^{\S}$ Supported by JSPS KAKENHI Grant Number 16K05262.
} 
One of the main streams of the study of the hamiltonian problem is, as mentioned above, to consider degree-sum-type sufficient conditions for graphs to have a Hamilton cycle. We list some of them below.

Theorem 1. Let $G$ be a graph of order at least three. If $G$ satisfies one of the following, then $G$ is hamiltonian.

(i) (Dirac [7]) The minimum degree of $G$ is at least $\frac{n}{2}$.

(ii) (Ore $[10]) \sigma_{2} \geqslant n$.

(iii) (Chvátal and Erdős [6]) $\alpha \leqslant \kappa$.

(iv) (Bondy [4]) G is k-connected and $\sigma_{k+1}>\frac{(k+1)(n-1)}{2}$.

(v) (Bauer, Broersma, Veldman and Li [2]) $G$ is 2-connected and $\sigma_{3} \geqslant n+\kappa$.

To be exact, Theorem 1 (iii) is not a degree-sum-type condition, but it is closely related. Bondy [3] showed that Theorem 1 (iii) implies (ii). From Theorem 1 (iii), it is natural to consider a $\sigma_{k+1}$ condition for a $k$-connected graph. Bondy [4] gave a $\sigma_{k+1}$ condition of Theorem 1 (iv).

In this paper, we give a much weaker $\sigma_{k+1}$ condition than that of Theorem 1 (iv).

Theorem 2. Let $k$ be a positive integer and let $G$ be a $k$-connected graph. If

$$
\sigma_{k+1} \geqslant n+\kappa+(k-2)(\alpha-1),
$$

then $G$ is hamiltonian.

Theorem 2 was conjectured by Ozeki and Yamashita [12]. The case $k=2$ of Theorem 2 coincides with Theorem $1(\mathrm{v})$. The cases $k=1$ and $k=3$ were shown by Fraisse and Jung [8], and by Ozeki and Yamashita [12], respectively.

\subsection{Sharpness of Theorem 2}

In this subsection, we show that the $\sigma_{k+1}$ condition in Theorem 2 is best possible in some senses.

We first discuss the lower bound of the $\sigma_{k+1}$ condition. For an integer $l \geqslant 2$ and $l$ vertex-disjoint graphs $H_{1}, \ldots, H_{l}$, we define the graph $H_{1} \vee \cdots \vee H_{l}$ from the union of $H_{1}, \ldots, H_{l}$ by joining every vertex of $H_{i}$ to every vertex of $H_{i+1}$ for $1 \leqslant i \leqslant l-1$. Fix a positive integer $k$. Let $\kappa, m$ and $n$ be integers with $k \leqslant \kappa<m$ and $2 m+1 \leqslant n \leqslant 3 m-\kappa$. Let $G_{1}=K_{n-2 m} \vee \bar{K}_{\kappa} \vee \bar{K}_{m} \vee \bar{K}_{m-\kappa}$ (see Figure 1), where $K_{p}$ denotes the complete graph of order $p$ and $\bar{K}_{p}$ denotes the complement of $K_{p}$. Then $\alpha\left(G_{1}\right)=m+1, \kappa\left(G_{1}\right)=\kappa$ and

$$
\sigma_{k+1}\left(G_{1}\right)=(n-2 m-1+\kappa)+k m=n\left(G_{1}\right)+\kappa\left(G_{1}\right)+(k-2)\left(\alpha\left(G_{1}\right)-1\right)-1 .
$$




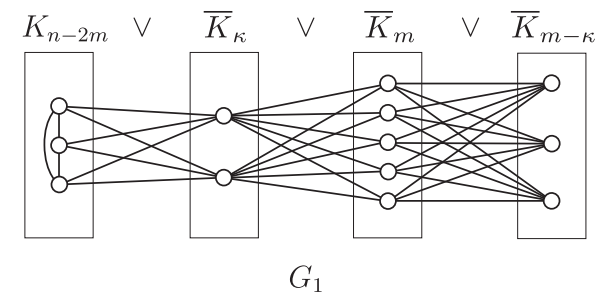

Figure 1: The case $n=13, m=5, \kappa=2$ of the graph $G_{1}$.

Since deleting all the vertices in $\bar{K}_{\kappa}$ and those in $\bar{K}_{m-\kappa}$ breaks $G_{1}$ into $m+1$ components, we see that $G_{1}$ has no Hamilton cycle. Therefore, the $\sigma_{k+1}$ condition in Theorem 2 is best possible.

We next discuss the relation between the coefficient of $\kappa$ and that of $\alpha-1$. By Theorem 1 (iii), we may assume that $\alpha \geqslant \kappa+1$. This implies that

$$
n+\kappa+(k-2)(\alpha-1) \geqslant n+(1+\varepsilon) \kappa+(k-2-\varepsilon)(\alpha-1)
$$

for arbitrarily $\varepsilon>0$. Then one may expect that the $\sigma_{k+1}$ condition in Theorem 2 can be replaced with " $n+(1+\varepsilon) \kappa+(k-2-\varepsilon)(\alpha-1)$ " for some $\varepsilon>0$. However, the graph $G_{1}$ as defined above shows that it is not true: For any $\varepsilon>0$, there exist two integers $m$ and $\kappa$ such that $\varepsilon(m-\kappa) \geqslant 1$. If we construct the above graph $G_{1}$ from such integers $m$ and $\kappa$, then we have

$$
\begin{aligned}
\sigma_{k+1}\left(G_{1}\right) & =n+(1+\varepsilon) \kappa+(k-2-\varepsilon) m-1+\varepsilon(m-\kappa) \\
& \geqslant n\left(G_{1}\right)+(1+\varepsilon) \kappa\left(G_{1}\right)+(k-2-\varepsilon)\left(\alpha\left(G_{1}\right)-1\right),
\end{aligned}
$$

but $G_{1}$ is not hamiltonian. This means that the coefficient 1 of $\kappa$ and the coefficient $k-2$ of $\alpha-1$ are, in a sense, best possible.

\subsection{Comparing Theorem 2 to other results}

In this subsection, we compare Theorem 2 to Theorem 1 (iv) and to Theorem 3 (see below).

We first show that the $\sigma_{k+1}$ condition of Theorem 2 is weaker than that of Theorem 1 (iv). Let $G$ be a $k$-connected graph satisfying the $\sigma_{k+1}$ condition of Theorem 1 (iv). Assume that $\alpha \geqslant(n+1) / 2$. Let $X$ be an independent set of order at least $(n+1) / 2$. Then $|V(G) \backslash X| \leqslant(n-1) / 2$ and $|V(G) \backslash X| \geqslant k$ since $V(G) \backslash X$ is a cut set. Hence $(n+1) / 2 \geqslant k+1$, and we can take a subset $Y$ of $X$ with $|Y|=k+1$. Then $N_{G}(y) \subseteq$ $V(G) \backslash X$ for $y \in Y$, and hence $\sum_{y \in Y} d_{G}(y) \leqslant(k+1)|V(G) \backslash X| \leqslant(k+1)(n-1) / 2$. This contradicts the $\sigma_{k+1}$ condition of Theorem 1 (iv). Therefore $n / 2 \geqslant \alpha$. Moreover, by Theorem 1 (iii), we may assume that $\alpha \geqslant \kappa+1$. Therefore, the following inequality holds:

$$
\begin{aligned}
\sigma_{k+1} & >\frac{(k+1)(n-1)}{2} \\
& \geqslant n-1+\frac{(k-1)(2 \alpha-1)}{2}
\end{aligned}
$$




$$
\begin{aligned}
& \geqslant n-1+(k-1)(\alpha-1) \\
& \geqslant n+\kappa+(k-2)(\alpha-1)-1 .
\end{aligned}
$$

Thus, the $\sigma_{k+1}$ condition of Theorem 1 (iv) implies that of Theorem 2 .

We next compare Theorem 2 to the following result of Ota.

Theorem 3 (Ota [11]). Let $G$ be a 2-connected graph. If $\sigma_{l+1} \geqslant n+l(l-1)$ for all integers $l$ with $l \geqslant \kappa$, then $G$ is hamiltonian.

We mention about the reason to compare Theorem 2 to Theorem 3. Li [9] proved the following theorem as a corollary of Theorem 3 .

Theorem 4 (Li [9]). Let $k$ be a positive integer and let $G$ be a $k$-connected graph. If $\sigma_{k+1} \geqslant n+(k-1)(\alpha-1)$, then $G$ is hamiltonian.

Note that Theorem 2 is, assuming Theorem 1 (iii), an improvement of Theorem 4 . Therefore we should show that Theorem 2 cannot be implied by Theorem 3 .

Let $\kappa, r, k, m$ be integers such that $4 \leqslant r, 3 \leqslant k \leqslant \kappa-2$ and $m=(k+1)(r-2)+4$. Let $G_{2}=K_{1} \vee \bar{K}_{\kappa} \vee K_{\kappa+m-r} \vee\left(\bar{K}_{m} \vee K_{r}\right)$. Then $n\left(G_{2}\right)=2 \kappa+2 m+1, \kappa\left(G_{2}\right)=\kappa$ and $\alpha\left(G_{2}\right)=\kappa+m$. Since

$$
\kappa+k(\kappa+m)-(k+1)(\kappa+m-r+1)=(k+1)(r-1)-m=k-3 \geqslant 0,
$$

it follows that

$$
\begin{aligned}
\sigma_{k+1}\left(G_{2}\right) & =\min \{\kappa+k(\kappa+m),(k+1)(\kappa+m-r+1)\} \\
& =\kappa+k(\kappa+m)-(k-3) \\
& =(2 \kappa+2 m+1)+\kappa+(k-2)(\kappa+m-1) \\
& =n\left(G_{2}\right)+\kappa\left(G_{2}\right)+(k-2)\left(\alpha\left(G_{2}\right)-1\right) .
\end{aligned}
$$

Hence the assumption of Theorem 2 holds. On the other hand, for $l=\alpha\left(G_{2}\right)-1=$ $\kappa+m-1$, we have

$$
\begin{aligned}
n\left(G_{2}\right)+l(l-1)-\sigma_{l+1}\left(G_{2}\right)= & (2 \kappa+2 m+1)+(\kappa+m-1)(\kappa+m-2) \\
& -\{\kappa(\kappa+m-r+1)+m(\kappa+m)\} \\
= & \kappa(r-2)-m+3 \\
= & (\kappa-k-1)(r-2)-1>0 .
\end{aligned}
$$

Hence the assumption of Theorem 3 does not hold. These yield that for the graph $G_{2}$, we can apply Theorem 2, but cannot apply Theorem 3. 


\section{Notation and lemmas}

Let $G$ be a graph and $H$ be a subgraph of $G$, and let $x \in V(G)$ and $X \subseteq V(G)$. We denote by $N_{G}(X)$ the set of vertices in $V(G) \backslash X$ which are adjacent to some vertex in $X$. We define $N_{H}(x):=N_{G}(x) \cap V(H)$ and $d_{H}(x):=\left|N_{H}(x)\right|$. Furthermore, we define $N_{H}(X):=N_{G}(X) \cap V(H)$. We denote by $G[X]$ the subgraph of $G$ induced by $X$, and let $G-X:=G[V(G) \backslash X]$. If there is no fear of confusion, we often identify $H$ with its vertex set $V(H)$. For example, we often write $G-H, G[H]$ and $X \cap H$ instead of $G-V(H), G[V(H)]$ and $X \cap V(H)$. A path $P$ is called an $H$-path if both end vertices of $P$ are contained in $H$ and all internal vertices are not contained in $H$. Note that each edge of $H$ is an $H$-path. The union of two vertex-disjoint graphs $H_{1}$ and $H_{2}$ is denoted by $H_{1} \cup H_{2}$.

Throughout this paper, we consider that each of cycles and paths has a fixed orientation. Let $C$ be a cycle (or a path) in a graph $G$. For $x, y \in V(C)$, we denote by $C[x, y]$ the path from $x$ to $y$ along the orientation of $C$. The reverse sequence of $C[x, y]$ is denoted by $\overleftarrow{C}[y, x]$. We denote $C[x, y]-\{x, y\}, C[x, y]-\{x\}$ and $C[x, y]-\{y\}$ by $C(x, y), C(x, y]$ and $C[x, y)$, respectively. We denote a path $P$ from a vertex $u$ to a vertex $v$ by $P[u, v]$. For two vertex-disjoint paths $P[u, v]$ and $Q[x, y]$, if $v=x$ or $v x \in E(G)$, then $P[u, v] Q[x, y]$ is the path from $u$ to $y$ along $P$ and $Q$. For $x \in V(C)$, we denote the successor and the predecessor of $x$ on $C$ by $x^{+}$and $x^{-}$, respectively. (For the end vertices $u, v$ of $P[u, v], u^{-}$and $v^{+}$do not exist.) For $X \subseteq V(C)$, we define $X^{+}:=\left\{x^{+}: x \in X\right\}$ and $X^{-}:=\left\{x^{-}: x \in X\right\}$.

In this paper, we extend the concept of insertible, introduced by Ainouche [1], which has been used for the proofs of the results on cycles. Let $G$ be a graph, and $H$ be a subgraph of $G$. Let $X(H):=\{x \in V(G-H): x u, x v \in E(G)$ for some $u v \in E(H)\}$, and for $x \in V(G-H)$, let $I(x ; H):=\{u v \in E(H): x u, x v \in E(G)\}$. Let $Y(H):=\{y \in$ $\left.V(G-H): d_{H}(y) \geqslant \alpha(G)\right\}$.

Lemma 5. Let $D$ be a cycle of a graph $G$. Let $Q_{1}, Q_{2}, \ldots, Q_{k}$ be vertex-disjoint paths in $G-D$, where $Q_{i}$ is a path from $a_{i}$ to $b_{i}, 1 \leqslant i \leqslant k$, and let $Q:=\bigcup_{i=1}^{k} Q_{i}$. If the following (I) and (II) hold, then $G[D \cup Q]$ is hamiltonian.

(I) $u \in X(D) \cup Y\left(Q_{i}\left(u, b_{i}\right] \cup D\right)$ for $u \in V\left(Q_{i}\right), 1 \leqslant i \leqslant k$.

(II) $I(x ; D) \cap I(y ; D)=\emptyset$ for $x \in V\left(Q_{i}\right)$ and $y \in V\left(Q_{j}\right), 1 \leqslant i<j \leqslant k$.

Proof. We can easily see that $G[D \cup Q]$ contains a cycle $D^{*}$ such that $V(D) \cup(X(D) \cap$ $Q) \subseteq V\left(D^{*}\right)$. In fact, we can insert all vertices of $X(D) \cap Q_{1}$ into $D$ by choosing the following $u_{1}, v_{1} \in V\left(Q_{1}\right)$ and $w_{1} w_{1}^{+} \in E(D)$ inductively. Take the first vertex $u_{1}$ in $X(D) \cap Q_{1}$ along the orientation of $Q_{1}$, and let $v_{1}$ be the last vertex in $X(D) \cap Q_{1}$ such that $I\left(u_{1} ; D\right) \cap I\left(v_{1} ; D\right) \neq \emptyset$. Then we can insert all vertices of $Q_{1}\left[u_{1}, v_{1}\right]$ into $D$. To be exact, taking $w_{1} w_{1}^{+} \in I\left(u_{1} ; D\right) \cap I\left(v_{1} ; D\right), D_{1}^{1}:=w_{1} Q_{1}\left[u_{1}, v_{1}\right] D\left[w_{1}^{+}, w_{1}\right]$ is such a cycle. By the choice of $u_{1}$ and $v_{1}, w_{1} w_{1}^{+} \notin I(x ; D)$ for all $x \in V\left(Q_{1}-Q_{1}\left[u_{1}, v_{1}\right]\right)$, and all vertices in $X(D) \cap V\left(Q_{1}-Q_{1}\left[u_{1}, v_{1}\right]\right)$ are contained in the path $Q_{1}-Q_{1}\left[a_{1}, v_{1}\right]$. Moreover, note that 
$E(D) \backslash\left\{w_{1} w_{1}^{+}\right\} \subseteq E\left(D_{1}^{1}\right)$. Hence by repeating this argument, we can obtain a cycle $D_{1}^{*}$ of $G\left[D \cup Q_{1}\right]$ such that $V(D) \cup\left(X(D) \cap Q_{1}\right) \subseteq V\left(D_{1}^{*}\right)$ and $E(D) \backslash \bigcup_{x \in V\left(Q_{1}\right)} I(x ; D) \subseteq E\left(D_{1}^{*}\right)$. Then by (II), $I(x ; D) \subseteq E\left(D_{1}^{*}\right)$ for all $x \in V(Q) \backslash V\left(Q_{1}\right)$. Therefore $G[D \cup Q]$ contains a cycle $D^{*}$ such that $V(D) \cup(X(D) \cap Q) \subseteq V\left(D^{*}\right)$.

We choose a cycle $C$ of $G[D \cup Q]$ containing all vertices in $V(D) \cup(X(D) \cap Q)$ so that $|C|$ is as large as possible. Now, we change the "base" cycle from $D$ to $C$, and use the symbol $(\cdot)^{+}$for the orientation of $C$. Suppose that $V\left(Q_{i}-C\right) \neq \emptyset$ for some $i, 1 \leqslant i \leqslant k$. We may assume that $i=1$. Let $w$ be the last vertex in $V\left(Q_{1}-C\right)$ along $Q_{1}$. Since $C$ contains all vertices in $X(D) \cap Q_{1}$, it follows from (I) that $w \in Y\left(Q_{1}\left(w, b_{1}\right] \cup D\right)$, that is, $\left|N_{G}(w) \cap\left(Q_{1}\left(w, b_{1}\right] \cup D\right)\right| \geqslant \alpha(G)$. By the choice of $w$, we obtain $V\left(Q_{1}\left(w, b_{1}\right] \cup D\right) \subseteq V(C)$. Therefore $\left|N_{C}(w)^{+} \cup\{w\}\right| \geqslant\left|N_{G}(w) \cap\left(Q_{1}\left(w, b_{1}\right] \cup D\right)\right|+1 \geqslant \alpha(G)+1$. This implies that $N_{C}(w)^{+} \cup\{w\}$ is not an independent set in $G$. Hence $w z^{+} \in E(G)$ for some $z \in N_{C}(w)$ or $z_{1}^{+} z_{2}^{+} \in E(G)$ for some distinct $z_{1}, z_{2} \in N_{C}(w)$. In the former case, let $C^{\prime}=w C\left[z^{+}, z\right] w$, and in the latter case, let $C^{\prime}:=w \overleftarrow{C}\left[z_{1}, z_{2}^{+}\right] C\left[z_{1}^{+}, z_{2}\right] w$. Then $C^{\prime}$ is a cycle of $G[D \cup Q]$ such that $V(C) \cup\{w\}=V\left(C^{\prime}\right)$, which contradicts the choice of $C$. Thus $V(Q)$ is contained in $C$, and hence $C$ is a Hamilton cycle of $G[D \cup Q]$.

In the rest of this section, we fix the following notation. Let $C$ be a longest cycle in a graph $G$, and $H_{0}$ be a component of $G-C$. For $u \in N_{C}\left(H_{0}\right)$, let $u^{\prime} \in N_{C}\left(H_{0}\right) \backslash\{u\}$ be a vertex such that $C\left(u, u^{\prime}\right) \cap N_{C}\left(H_{0}\right)=\emptyset$, that is, $u^{\prime}$ is the successor of $u$ in $N_{C}\left(H_{0}\right)$ along the orientation of $C$.

For $u \in N_{C}\left(H_{0}\right)$, a vertex $v \in C\left(u, u^{\prime}\right)$ is insertible if $v \in X\left(C\left[u^{\prime}, u\right]\right) \cup Y(C(v, u])$. A vertex in $C\left(u, u^{\prime}\right)$ is said to be non-insertible if it is not insertible.

Lemma 6. There exists a non-insertible vertex in $C\left(u, u^{\prime}\right)$ for $u \in N_{C}\left(H_{0}\right)$.

Proof. Let $u \in N_{C}\left(H_{0}\right)$, and suppose that every vertex in $C\left(u, u^{\prime}\right)$ is insertible. Let $P$ be a $C$-path joining $u$ and $u^{\prime}$ with $V(P) \cap V\left(H_{0}\right) \neq \emptyset$. Let $D:=C\left[u^{\prime}, u\right] P\left[u, u^{\prime}\right]$ and $Q:=$ $C\left(u, u^{\prime}\right)$. Let $v \in V(Q)$. Since $v$ is insertible, it follows that $v \in X\left(C\left[u^{\prime}, u\right]\right) \cup Y(C(v, u])$. Since $C\left[u^{\prime}, u\right]$ is a subpath of $D$, we have $v \in X(D) \cup Y\left(Q\left(v, u^{\prime}\right) \cup D\right)$. Hence, by Lemma $5, G[D \cup Q]$ is hamiltonian, which contradicts the maximality of $C$.

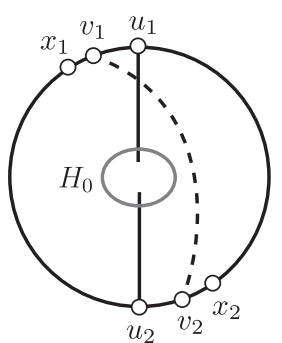

(i)

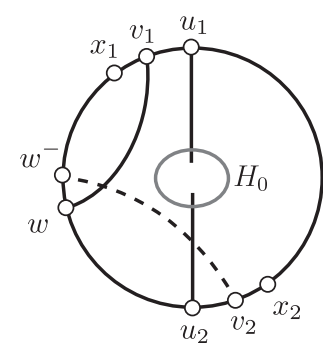

(ii)

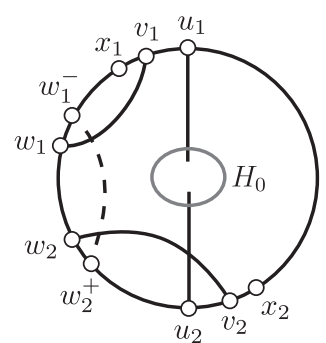

(iii)

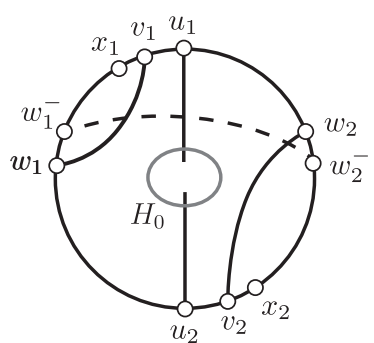

(iv)

Figure 2: Lemma 7. 
Lemma 7. Let $u_{1}, u_{2} \in N_{C}\left(H_{0}\right)$ with $u_{1} \neq u_{2}$, and let $x_{i}$ be the first non-insertible vertex along $C\left(u_{i}, u_{i}^{\prime}\right)$ for $i=1,2$. Then the following hold (see Figure 2).

(i) There exists no $C$-path joining $v_{1} \in C\left(u_{1}, x_{1}\right]$ and $v_{2} \in C\left(u_{2}, x_{2}\right]$. In particular, $x_{1} x_{2} \notin E(G)$.

(ii) If there exists a $C$-path joining $v_{1} \in C\left(u_{1}, x_{1}\right]$ and $w \in C\left(v_{1}, u_{2}\right]$, then there exists no $C$-path joining $v_{2} \in C\left(u_{2}, x_{2}\right]$ and $w^{-}$.

(iii) If there exist a $C$-path joining $v_{1} \in C\left(u_{1}, x_{1}\right]$ and $w_{1} \in C\left(v_{1}, u_{2}\right)$ and a $C$-path joining $v_{2} \in C\left(u_{2}, x_{2}\right]$ and $w_{2} \in C\left[w_{1}, u_{2}\right)$, then there exists no $C$-path joining $w_{1}^{-}$ and $w_{2}^{+}$.

(iv) If for each $i=1,2$, there exists a $C$-path joining $v_{i} \in C\left(u_{i}, x_{i}\right]$ and $w_{i} \in C\left(v_{i}, u_{3-i}\right]$, then there exists no $C$-path joining $w_{1}^{-}$and $w_{2}^{-}$.

Proof. Let $P_{0}$ be a $C$-path which connects $u_{1}$ and $u_{2}$, and $V\left(P_{0}\right) \cap V\left(H_{0}\right) \neq \emptyset$. We first show (i) and (ii). Suppose that one of the following holds for some $v_{1} \in C\left(u_{1}, x_{1}\right]$ and some $v_{2} \in C\left(u_{2}, x_{2}\right]$ : (a) There exists a $C$-path $P_{1}$ joining $v_{1}$ and $v_{2}$. (b) There exist disjoint $C$-paths $P_{2}$ joining $v_{l}$ and $w$, and $P_{3}$ joining $v_{3-l}$ and $w^{-}$for some $l=1,2$ and some $w \in C\left(v_{l}, u_{3-l}\right]$. We choose such vertices $v_{1}$ and $v_{2}$ so that $\left|C\left[u_{1}, v_{1}\right]\right|+\left|C\left[u_{2}, v_{2}\right]\right|$ is as small as possible. Without loss of generality, we may assume that $l=1$ if (b) holds. Since $N_{C}\left(H_{0}\right) \cap\left\{v_{1}, v_{2}\right\}=\emptyset,\left(V\left(P_{1}\right) \cup V\left(P_{2}\right) \cup V\left(P_{3}\right)\right) \cap V\left(P_{0}\right)=\emptyset$. Therefore, we can define a cycle

$$
D:= \begin{cases}P_{1}\left[v_{1}, v_{2}\right] C\left[v_{2}, u_{1}\right] P_{0}\left[u_{1}, u_{2}\right] \overleftarrow{C}\left[u_{2}, v_{1}\right] & \text { if (a) holds } \\ P_{2}\left[v_{1}, w\right] C\left[w, u_{2}\right] \overleftarrow{P_{0}}\left[u_{2}, u_{1}\right] \overleftarrow{C}\left[u_{1}, v_{2}\right] P_{3}\left[v_{2}, w^{-}\right] \overleftarrow{C}\left[w^{-}, v_{1}\right] & \text { if (b) holds }\end{cases}
$$

For $i=1,2$, let $Q_{i}:=C\left(u_{i}, v_{i}\right)$ (possibly $V\left(Q_{i}\right)=\emptyset$ ). By the choice of $x_{i}$ and Lemma 6 , we have $N_{C}\left(H_{0}\right) \cap Q_{i}=\emptyset$, and hence the following statement (1) holds. By the choice of $v_{1}$ and $v_{2}$, we can obtain the following statements (2)-(5).

(1) $N_{G}(x) \cap P_{0}\left(u_{1}, u_{2}\right)=\emptyset$ for $x \in V\left(Q_{1} \cup Q_{2}\right)$.

(2) $N_{G}(x) \cap\left(P_{1}\left(v_{1}, v_{2}\right) \cup P_{2}\left(v_{1}, w\right) \cup P_{3}\left(v_{2}, w^{-}\right)\right)=\emptyset$ for $x \in V\left(Q_{1} \cup Q_{2}\right)$.

(3) $x y \notin E(G)$ for $x \in V\left(Q_{1}\right)$ and $y \in V\left(Q_{2}\right)$.

(4) $I(x ; C) \cap I(y ; C)=\emptyset$ for $x \in V\left(Q_{1}\right)$ and $y \in V\left(Q_{2}\right)$.

(5) If (b) holds, then $w^{-} w \notin I(x ; C)$ for $x \in V\left(Q_{1} \cup Q_{2}\right)$.

Let $u \in V\left(Q_{i}\right)$ for some $i=1,2$. Note that each vertex of $Q_{i}$ is insertible, that is, $u \in$ $X\left(C\left[u_{i}^{\prime}, u_{i}\right]\right) \cup Y\left(C\left(u, u_{i}\right]\right)$. We show that $u \in X(D) \cup Y\left(Q_{i}\left(u, v_{i}\right) \cup D\right)$. If $u \in X\left(C\left[u_{i}^{\prime}, u_{i}\right]\right)$ and $V\left(Q_{3-i}\right) \neq \emptyset$, then the statements $(3)$ and (5) yield that $u \in X(D)$. If $u \in X\left(C\left[u_{i}^{\prime}, u_{i}\right]\right)$ and $V\left(Q_{3-i}\right)=\emptyset$, then the choice of $v_{1}$ and $v_{2}$ and the statement $(5)$ yield that $u \in X(D)$. Suppose that $u \in Y\left(C\left(u, u_{i}\right]\right)$. By $(3), N_{G}(u) \cap C\left(u, u_{i}\right] \subseteq N_{G}(u) \cap\left(Q_{i}\left(u, v_{i}\right) \cup D\right)$. This 
implies that $u \in Y\left(Q_{i}\left(u, v_{i}\right) \cup D\right)$. By $(1),(2)$ and $(4), I(x ; D) \cap I(y ; D)=\emptyset$ for $x \in V\left(Q_{1}\right)$ and $y \in V\left(Q_{2}\right)$. Thus, by Lemma $5, G\left[D \cup Q_{1} \cup Q_{2}\right]$ is hamiltonian, which contradicts the maximality of $C$.

By using a similar argument as above, we can also show (iii) and (iv). We only denote the outline of the proof of (iii). Suppose that for some $v_{1} \in C\left(u_{1}, x_{1}\right]$ and $v_{2} \in C\left(u_{2}, x_{2}\right]$, there exist disjoint $C$-paths $P_{1}\left[v_{1}, w_{1}\right], P_{2}\left[v_{2}, w_{2}\right]$ and $P_{3}\left[w_{1}^{-}, w_{2}^{+}\right]$with $w_{1} \in C\left(v_{1}, u_{2}\right)$ and $w_{2} \in C\left[w_{1}, u_{2}\right)$. We choose such $v_{1}$ and $v_{2}$ so that $\left|C\left[u_{1}, v_{1}\right]\right|+\left|C\left[u_{2}, v_{2}\right]\right|$ is as small as possible. Let $Q_{i}:=C\left(u_{i}, v_{i}\right)$ for $i=1,2$. Then by Lemma 7 (i), $x y \notin E(G)$ for $x \in V\left(Q_{1}\right)$ and $y \in V\left(Q_{2}\right)$. By the choice of $v_{1}$ and $v_{2}$ and Lemma 7 (ii), $w_{1} w_{1}^{-}, w_{2} w_{2}^{+} \notin$ $I\left(x ; C\left[v_{1}, u_{1}\right]\right) \cup I\left(y ; C\left[v_{2}, u_{2}\right]\right)$ for $x \in V\left(Q_{1}\right)$ and $y \in V\left(Q_{2}\right)$. By Lemma 7 (i) and (ii), $I\left(x ; C\left[v_{1}, u_{2}\right] \cup C\left[v_{2}, u_{1}\right]\right) \cap I\left(y ; C\left[v_{1}, u_{2}\right] \cup C\left[v_{2}, u_{1}\right]\right)=\emptyset$ for $x \in V\left(Q_{1}\right)$ and $y \in V\left(Q_{2}\right)$. Hence by applying Lemma 5 as

$$
D:=P_{1}\left[v_{1}, w_{1}\right] C\left[w_{1}, w_{2}\right] \overleftarrow{P_{2}}\left[w_{2}, v_{2}\right] C\left[v_{2}, u_{1}\right] P_{0}\left[u_{1}, u_{2}\right] \overleftarrow{C}\left[u_{2}, w_{2}^{+}\right] \overleftarrow{P_{3}}\left[w_{2}^{+}, w_{1}^{-}\right] \overleftarrow{C}\left[w_{1}^{-}, v_{1}\right]
$$

$Q_{1}$ and $Q_{2}$, we see that there exits a longer cycle than $C$, a contradiction.

\section{Proof of Theorem 2}

Proof of Theorem 2. The cases $k=1, k=2$ and $k=3$ were shown by Fraisse and Jung [8], by Bauer et al. [2] and by Ozeki and Yamashita [12], respectively. Therefore, we may assume that $k \geqslant 4$. Let $G$ be a graph satisfying the assumption of Theorem 2 . By Theorem 1 (iii), we may assume $\alpha(G) \geqslant \kappa(G)+1$. Let $C$ be a longest cycle in $G$. If $C$ is a Hamilton cycle of $G$, then there is nothing to prove. Hence we may assume that $G-V(C) \neq \emptyset$. Set $H:=G-V(C)$ and $x_{0} \in V(H)$. Choose a longest cycle $C$ and $x_{0}$ so that

$$
d_{C}\left(x_{0}\right) \text { is as large as possible. }
$$

Let $H_{0}$ be the component of $H$ such that $x_{0} \in V\left(H_{0}\right)$. Set

$$
U:=N_{C}\left(H_{0}\right):=\left\{u_{1}, u_{2}, \ldots, u_{m}\right\} .
$$

Note that $m \geqslant \kappa(G) \geqslant k$. Let $u_{i}^{\prime}$ be the vertex in $N_{C}\left(H_{0}\right) \backslash\left\{u_{i}\right\}$ such that $C\left(u_{i}, u_{i}^{\prime}\right) \cap$ $N_{C}\left(H_{0}\right)=\emptyset$ for each $i \in[m]$, where $[m]$ means $\{1,2, \ldots, m\}$. By Lemma 6 , there exists a non-insertible vertex in $C\left(u_{i}, u_{i}^{\prime}\right)$. Let $x_{i} \in C\left(u_{i}, u_{i}^{\prime}\right)$ be the first non-insertible vertex along the orientation of $C$ for each $i \in[m]$, and set

$$
X:=\left\{x_{1}, x_{2}, \ldots, x_{m}\right\} .
$$

By Lemma 7 (i) and since $N_{H_{0}}\left(x_{i}\right)=\emptyset$ for $i \in[m]$, we obtain the following.

Claim 8. $X \cup\left\{x_{0}\right\}$ is an independent set, and hence $|X| \leqslant \alpha(G)-1$.

Define

$$
D_{0}:=\emptyset \quad \text { and } \quad D_{i}:=C\left(u_{i}, x_{i}\right) \quad \text { for each } i \in[m]
$$


and $D:=\bigcup_{i \in[m]} D_{i}$. By Claim 8 and the definition of $U$ and $X$, we obtain

$$
d_{C}\left(x_{0}\right) \leqslant|U|=|X| \leqslant \alpha(G)-1 .
$$

Since $D_{0}=\emptyset$ and $x_{i}$ is non-insertible, we can see that

$$
d_{C}\left(x_{i}\right) \leqslant\left|D_{i}\right|+\alpha(G)-1 \quad \text { for } 0 \leqslant i \leqslant m .
$$

By the definition of $x_{i}, N_{H_{0}}\left(x_{i}\right)=\emptyset$ for $i \in[m]$. By Lemma 7 (i), $N_{H}\left(x_{i}\right) \cap N_{H}\left(x_{j}\right)=\emptyset$ for $i, j \in[m]$ with $i \neq j$. Thus we obtain

$$
\sum_{0 \leqslant i \leqslant m} d_{H}\left(x_{i}\right) \leqslant|H|-1
$$

In this paragraph, let $i$ and $j$ be distinct two integers in $[m]$, and set $C_{i}:=C\left[x_{i}, u_{j}\right]$ and $C_{j}:=C\left[x_{j}, u_{i}\right]$. By Lemma 7 (ii), we have $N_{C_{i}}\left(x_{i}\right)^{-} \cap N_{C_{i}}\left(x_{j}\right)=\emptyset$ and $N_{C_{j}}\left(x_{j}\right)^{-} \cap$ $N_{C_{j}}\left(x_{i}\right)=\emptyset$. By Lemma 7 (i), $N_{C_{i}}\left(x_{i}\right)^{-} \cup N_{C_{i}}\left(x_{j}\right) \subseteq C_{i} \backslash D, N_{C_{j}}\left(x_{j}\right)^{-} \cup N_{C_{j}}\left(x_{i}\right) \subseteq C_{j} \backslash D$ and $N_{D_{i}}\left(x_{j}\right)=N_{D_{j}}\left(x_{i}\right)=\emptyset$. Thus, we obtain

$$
d_{C}\left(x_{i}\right)+d_{C}\left(x_{j}\right) \leqslant|C|-\sum_{h \in[m] \backslash\{i, j\}}\left|D_{h}\right| \quad \text { for } i, j \in[m] \text { with } i \neq j .
$$

We will frequently use these upper bounds (1)-(4) on degree (sum) of vertices in $X \cup\left\{x_{0}\right\}$.

By replacing the labels $x_{2}$ and $x_{3}$ if necessary, we may assume that $x_{1}, x_{2}$ and $x_{3}$ appear in this order along the orientation of $C$. From this paragraph to the paragraph below Claim 9, the indices are taken modulo 3. From now on, for each $i \in[3]$, set

$$
C_{i}:=C\left[x_{i}, u_{i+1}\right]
$$

and

$$
W_{i}:=\left\{w \in V\left(C_{i}\right): w^{+} \in N_{C_{i}}\left(x_{i}\right) \text { and } w^{-} \in N_{C_{i}}\left(x_{i+1}\right)\right\}
$$

and $W:=W_{1} \cup W_{2} \cup W_{3}$ (see Figure 3 (i)). Note that $W \cap\left(U \cup\left\{x_{1}, x_{2}, x_{3}\right\}\right)=\emptyset$, by the definition of $C_{i}$ and $W_{i}$ and by Lemma 7 (i). Furthermore, for $i \in[3]$, set

$$
L_{i}:=\left\{x_{j} \in X \backslash\left\{x_{i+1}\right\}: N_{C_{i}}\left(x_{i+1}\right)^{+} \cap D_{j} \neq \emptyset\right\}
$$

and $L:=L_{1} \cup L_{2} \cup L_{3}$ (see Figure 3 (ii)). By the definition and Lemma 7 (i),

$$
W \cap L=\emptyset .
$$

In the following proof, we will set suitable three vertices as $x_{1}, x_{2}, x_{3}$ if necessary. Note that $W$ and $L$ will be defined by them in each case. Moreover, note that the following claims which hold for $x_{1}, x_{2}, x_{3}$, in fact, hold for any $x_{i}, x_{j}, x_{k}$ with respect to corresponding $W$ and $L$.

By Lemma 7, we can improve Claim 8 as follows: 


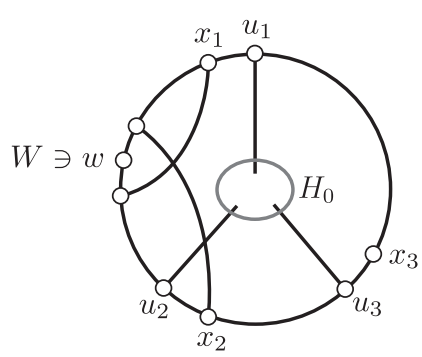

(i)

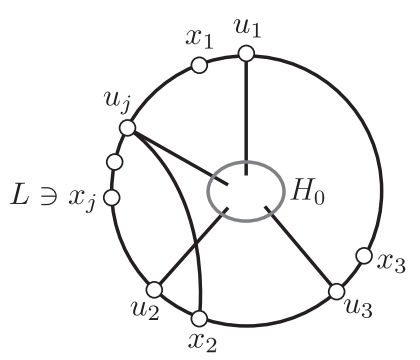

(ii)

Figure 3: The definition of $W$ and $L$.

Claim 9. $X \cup W \cup\left\{x_{0}\right\}$ is an independent set.

We now check the upper bound of $d_{C}\left(x_{1}\right)+d_{C}\left(x_{2}\right)+d_{C}\left(x_{3}\right)$. By Lemma 7(ii), $\left(N_{C_{i}}\left(x_{i}\right)^{-} \cup N_{C_{i}}\left(x_{i+1}\right)^{+}\right) \cap N_{C_{i}}\left(x_{i+2}\right)=\emptyset$ for each $i \in[3]$. Clearly, $N_{C_{i}}\left(x_{i}\right)^{-} \cap N_{C_{i}}\left(x_{i+1}\right)^{+}=$ $W_{i}$ and $N_{C_{i}}\left(x_{i}\right)^{-} \cup N_{C_{i}}\left(x_{i+1}\right)^{+} \cup N_{C_{i}}\left(x_{i+2}\right) \subseteq C_{i} \cup\left\{u_{i+1}^{+}\right\}$. By Lemma $7(\mathrm{i}),\left(N_{C_{i}}\left(x_{i}\right)^{-} \cup\right.$ $\left.N_{C_{i}}\left(x_{i+2}\right)\right) \cap D_{j}=\emptyset$ for each $i \in[3]$ and $j \in[m]$. Note that

$$
\left|N_{C_{i}}\left(x_{i+1}\right)^{+} \cap\left(\bigcup_{j \in[m] \backslash\{i+1\}} D_{j}\right)\right|=\left|L_{i}\right|
$$

for $i \in[3]$. Note also that $L \cap\left\{x_{1}, x_{2}, x_{3}\right\}=\emptyset$ and $W \cap L=\emptyset$ by (5). Therefore, for $i \in[3]$, the following inequality holds:

$$
\begin{aligned}
& d_{C_{i}}\left(x_{1}\right)+d_{C_{i}}\left(x_{2}\right)+d_{C_{i}}\left(x_{3}\right) \\
& =\left|N_{C_{i}}\left(x_{i}\right)^{-} \cup N_{C_{i}}\left(x_{i+1}\right)^{+} \cup N_{C_{i}}\left(x_{i+2}\right)\right| \\
& \quad+\left|\left(N_{C_{i}}\left(x_{i}\right)^{-} \cup N_{C_{i}}\left(x_{i+1}\right)^{+}\right) \cap N_{C_{i}}\left(x_{i+2}\right)\right|+\left|N_{C_{i}}\left(x_{i}\right)^{-} \cap N_{C_{i}}\left(x_{i+1}\right)^{+}\right| \\
& \leqslant\left|C_{i}\right|+\left|W_{i}\right|+1-\sum_{j \in[m]}\left|C_{i} \cap D_{j}\right|+\left|L_{i}\right| .
\end{aligned}
$$

By Lemma 7 (i), we have $N_{C}\left(x_{i}\right) \cap D_{j}=\emptyset$ for $i, j \in[m], i \neq j$, and hence

$$
d_{D_{i}}\left(x_{1}\right)+d_{D_{i}}\left(x_{2}\right)+d_{D_{i}}\left(x_{3}\right) \leqslant\left|D_{i}\right|
$$

for $i \in[3]$. Let $I$ be a subset of $\{0,1, \ldots, m\} \backslash\{1,2,3\}$ and let $L_{I}:=L \cap\left\{x_{i}: i \in I\right\}$ (We will set a suitable subset $I$ for each case.). Note that $\left|L \cap\left\{x_{i}\right\}\right|-\left|D_{i}\right| \leqslant 0$ for each $i \in[m] \backslash[3]$. Thus, we deduce

$$
\begin{aligned}
d_{C}\left(x_{1}\right)+d_{C}\left(x_{2}\right)+d_{C}\left(x_{3}\right) & \leqslant \sum_{i=1}^{3}\left(\left|C_{i}\right|+\left|W_{i}\right|+\left|L_{i}\right|+1-\sum_{j \in[m]}\left|C_{i} \cap D_{j}\right|+\left|D_{i}\right|\right) \\
& =|C|+|W|+|L|-\sum_{i \in[m] \backslash[3]}\left|D_{i}\right|+3 \\
& \leqslant|C|+|W|+\left|L_{I}\right|-\sum_{i \in I}\left|D_{i}\right|+3 .
\end{aligned}
$$


Claim 10. $|W \cup L| \geqslant \kappa(G)-2 \geqslant 2$.

Proof. Let $I$ be a $(k-2)$-subset of $\{0,1, \ldots, m\} \backslash\{1,2,3\}$, where a $k$-subset is a subset of order $k$. Suppose that $|W|+\left|L_{I}\right| \leqslant \kappa(G)-3$. By Claim 8, $\left\{x_{i}: i \in I\right\} \cup\left\{x_{1}, x_{2}, x_{3}\right\}$ is independent. By (6), we obtain

$$
d_{C}\left(x_{1}\right)+d_{C}\left(x_{2}\right)+d_{C}\left(x_{3}\right) \leqslant|C|+\kappa(G)-\sum_{i \in I}\left|D_{i}\right| .
$$

Therefore, this inequality and (2) and (3) yield that

$$
\sum_{i=1}^{3} d_{G}\left(x_{i}\right)+\sum_{i \in I} d_{G}\left(x_{i}\right) \leqslant n+\kappa(G)+(k-2)(\alpha(G)-1)-1,
$$

a contradiction. Therefore, by (5) and since $\kappa(G) \geqslant k \geqslant 4$, we obtain $|W \cup L|=$ $|W|+|L| \geqslant|W|+\left|L_{I}\right| \geqslant \kappa(G)-2 \geqslant 2$.

Claim 11. $|X| \geqslant \kappa(G)+1$.

Proof. Let $s$ and $t$ be distinct two integers in $[m]$. By (4), we have

$$
d_{C}\left(x_{s}\right)+d_{C}\left(x_{t}\right) \leqslant|C|-\sum_{i \in[m] \backslash\{s, t\}}\left|D_{i}\right| .
$$

Let $I$ be a $(k+1)$-subset of $\{0,1, \ldots, m\}$ such that $\{0, s, t\} \subseteq I$. By Claim $8,\left\{x_{i}: i \in I\right\}$ is an independent set. By (2), we deduce

$$
\sum_{i \in I \backslash\{0, s, t\}} d_{C}\left(x_{i}\right) \leqslant \sum_{i \in I \backslash\{0, s, t\}}\left|D_{i}\right|+(k-2)(\alpha(G)-1) .
$$

By (3), we obtain $\sum_{i \in I} d_{H}\left(x_{i}\right) \leqslant|H|-1$. Thus, it follows from the above three inequalities that

$$
\sum_{i \in I} d_{G}\left(x_{i}\right) \leqslant n+(k-2)(\alpha(G)-1)-1+d_{C}\left(x_{0}\right) .
$$

Since $\sigma_{k+1}(G) \geqslant n+\kappa(G)+(k-2)(\alpha(G)-1)$, we have $|X| \geqslant d_{C}\left(x_{0}\right) \geqslant \kappa(G)+1$.

Let $S$ be a cut set with $|S|=\kappa(G)$. By Claim 11, there exists an integer $l \in[m]$ such that $C\left[u_{l}, u_{l}^{\prime}\right) \cap S=\emptyset$. Hence all vertices in $C\left[u_{l}, u_{l}^{\prime}\right)$ are contained in some component of $G-S$. Let

$$
V_{1} \text { be the component of } G-S \text { such that } C\left[u_{l}, u_{l}^{\prime}\right) \subseteq V_{1}
$$

and

$$
V_{2}:=G-\left(S \cup V_{1}\right)
$$

By Lemma 7 (i), we obtain

$$
d_{C}\left(x_{l}\right) \leqslant\left|C \cap\left(V_{1} \cup S\right)\right|-\left|\bigcup_{i \in[m] \backslash\{l\}} D_{i} \cap\left(V_{1} \cup S\right)\right|-\left|X \cap\left(V_{1} \cup S\right)\right| .
$$


Claim 12. $D \cup X \cup W \cup H \subseteq V_{1} \cup S$. In particular, $x_{0} \in V_{1} \cup S$.

Proof. We first show that $D \cup X \cup W \subseteq V_{1} \cup S$. Suppose not. Then, for some $h \in[m] \backslash\{l\}$, there exists a vertex $v \in\left(D_{h} \cup\left\{x_{h}\right\} \cup\left(W \cap C\left(x_{h}, u_{h}^{\prime}\right)\right)\right) \cap V_{2}$. Choose $v$ so that $v=x_{h}$ if possible. Note that if $v \notin D_{h}$ then $x_{h} \notin N_{G}(v)$ by Lemma 7 (ii); if $v \in D_{h}$ and $x_{h} \in N_{G}(v)$ then $x_{h} \in S$ by the choice of $v$. Since $v \in V_{2}$, it follows from Lemma 7 (i) and (ii) that

$$
d_{C}(v) \leqslant\left|C \cap\left(V_{2} \cup S\right)\right|-\left|\bigcup_{i \in[m] \backslash\{h\}} D_{i} \cap\left(V_{2} \cup S\right)\right|-\left|X \cap\left(V_{2} \cup S\right)\right|+\left|\left\{x_{h}\right\} \cap S\right| .
$$

Let $I$ be a $k$-subset of $\{0,1, \ldots, m\} \backslash\{h\}$ such that $\{0, l\} \subseteq I$. By Claim 8 and Lemma 7 (i) and (ii), $\left\{x_{i}: i \in I\right\} \cup\{v\}$ is independent. By the above inequality and (1) and (7), we obtain

$$
\begin{aligned}
d_{C}\left(x_{l}\right)+d_{C}(v) \leqslant & \left|C \cap\left(V_{1} \cup V_{2} \cup S\right)\right|+|C \cap S|-\left|\bigcup_{i \in[m] \backslash\{l, h\}} D_{i} \cap\left(V_{1} \cup V_{2} \cup S\right)\right| \\
& -\left|X \cap\left(V_{1} \cup V_{2} \cup S\right)\right|-|X \cap S|+\left|\left\{x_{h}\right\} \cap S\right| \\
\leqslant & |C|+|C \cap S|-\sum_{i \in[m] \backslash\{l, h\}}\left|D_{i}\right|-|X| \\
\leqslant & |C|+\kappa(G)-\sum_{i \in I \backslash\{0, l\}}\left|D_{i}\right|-d_{C}\left(x_{0}\right) .
\end{aligned}
$$

On the other hand, (2) yields that

$$
\sum_{i \in I \backslash\{0, l\}} d_{C}\left(x_{i}\right) \leqslant \sum_{i \in I \backslash\{0, l\}}\left|D_{i}\right|+(k-2)(\alpha(G)-1) .
$$

By the above two inequalities, we deduce

$$
\sum_{i \in I} d_{C}\left(x_{i}\right)+d_{C}(v) \leqslant|C|+\kappa(G)+(k-2)(\alpha(G)-1) .
$$

Recall that $\left\{x_{i}: i \in I\right\} \cup\{v\}$ is independent, in particular, $x_{0} \notin \bigcup_{i \in I} N_{H}\left(x_{i}\right) \cup N_{H}(v)$. Since $N_{H}\left(x_{i}\right) \cap N_{H}\left(x_{j}\right)=\emptyset$ for $i, j \in I$ with $i \neq j$ and $\left(\bigcup_{i \in I} N_{H}\left(x_{i}\right)\right) \cap N_{H}(v)=\emptyset$ by Lemma 7 (i) and (ii), it follows that $\sum_{i \in I} d_{H}\left(x_{i}\right)+d_{H}(v) \leqslant|H|-1$. Combining this inequality with the above inequality, we get $\sum_{i \in I} d_{G}\left(x_{i}\right)+d_{G}(v) \leqslant n+\kappa(G)+(k-$ 2) $(\alpha(G)-1)-1$, a contradiction.

We next show that $H-H_{0} \subseteq V_{1} \cup S$. Suppose not. Then, there exists a vertex $y \in$ $\left(H-H_{0}\right) \cap V_{2}$. Let $H_{y}$ be the component of $H$ with $y \in V\left(H_{y}\right)$. If $N_{C}\left(H_{y}\right) \cap\left(D_{h} \cup\left\{x_{h}\right\}\right) \neq \emptyset$ for some $h \in[m] \backslash\{l\}$, then let $M:=\{0,1, \ldots, m\} \backslash\{h\}$ and by Lemma 7 (i),

$$
d_{C}(y) \leqslant\left|C \cap\left(V_{2} \cup S\right)\right|-\left|\bigcup_{i \in[m] \backslash\{h\}} D_{i} \cap\left(V_{2} \cup S\right)\right|-\left|X \cap\left(V_{2} \cup S\right)\right|+\left|\left\{x_{h}\right\} \cap S\right| ;
$$


if $N_{C}\left(H_{y}\right) \cap\left(D_{i} \cup\left\{x_{i}\right\}\right)=\emptyset$ for all $i \in[m] \backslash\{l\}$, then let $M:=\{0,1, \ldots, m\}$ and by this assumption,

$$
d_{C}(y) \leqslant\left|C \cap\left(V_{2} \cup S\right)\right|-\left|\bigcup_{i \in[m]} D_{i} \cap\left(V_{2} \cup S\right)\right|-\left|X \cap\left(V_{2} \cup S\right)\right| .
$$

Let $I$ be a $k$-subset of $M$ such that $\{0, l\} \subseteq I$. By the same argument as above, we obtain

$$
\sum_{i \in I} d_{C}\left(x_{i}\right)+d_{C}(y) \leqslant|C|+|C \cap S|+(k-2)(\alpha(G)-1) .
$$

If $N_{C}\left(H_{y}\right) \cap\left(D_{h} \cup\left\{x_{h}\right\}\right) \neq \emptyset$ for some $h \in[m] \backslash\{l\}$, then Lemma 7 (i) and (ii) yield that $\left(\bigcup_{i \in I \backslash\{l\}} N_{H}\left(x_{i}\right)\right) \cap V\left(H_{y}\right)=\emptyset$; otherwise, since $H_{0} \neq H_{y}$ and $N_{C}\left(H_{y}\right) \cap\left(D_{i} \cup\left\{x_{i}\right\}\right)=\emptyset$ for all $i \in[m] \backslash\{l\}$, the same conclusion holds. In particular, $y \notin \bigcup_{i \in I \backslash\{l\}} N_{G}\left(x_{i}\right)$. Since $x_{l} \in V_{1}$ and $y \in V_{2}$, we have $x_{l} y \notin E(G)$ and $N_{H}\left(x_{l}\right) \cap N_{H}(y) \subseteq H \cap S$. Therefore, we obtain $\left\{x_{i}: i \in I\right\} \cup\{y\}$ is independent, and

$$
\sum_{i \in I} d_{H}\left(x_{i}\right)+d_{H}(y) \leqslant|H|+|H \cap S|-\left|\left\{x_{0}, y\right\}\right|=|H|+|H \cap S|-2 .
$$

Combining the above two inequalities, $\sum_{i \in I} d_{G}\left(x_{i}\right)+d_{G}(y) \leqslant n+\kappa(G)+(k-2)(\alpha(G)-$ 1) -2 , a contradiction.

We finally show that $H_{0} \subseteq V_{1} \cup S$. Suppose not, that is, there exists a vertex $y_{0} \in H_{0} \cap V_{2}$. Then

$$
d_{G}\left(y_{0}\right) \leqslant\left|U \cap\left(V_{2} \cup S\right)\right|+\left|H_{0}\right|-1 .
$$

Since $u_{l} \in V_{1}$, we have $H_{0} \cap S \neq \emptyset$. Note that by the above argument, $X \subseteq V_{1} \cup S$. Therefore, by Claim 11, $\left|X \cap V_{1}\right|=|X|-|X \cap S| \geqslant \kappa(G)+1-\left(|S|-\left|H_{0} \cap S\right|\right) \geqslant$ $\kappa(G)+1-(\kappa(G)-1)=2$. Hence there exists a vertex $x_{s} \in\left(X \cap V_{1}\right) \backslash\left\{x_{l}\right\}$. Let $I$ be a $k$-subset of $[m]$ such that $\{l, s\} \subseteq I$. Then $\left\{x_{i}: i \in I\right\} \cup\left\{y_{0}\right\}$ is an independent set of order $k+1$. By Lemma 7 (i), we have $N_{C}\left(x_{l}\right)^{-} \cap\left(U \backslash\left\{u_{l}\right\}\right)=\emptyset$ and $N_{C}\left(x_{s}\right)^{-} \cap\left(U \backslash\left\{u_{s}\right\}\right)=\emptyset$. Since $\left\{x_{l}, x_{s}\right\} \subseteq V_{1}$, it follows that $\left(N_{C}\left(x_{l}\right) \cup N_{C}\left(x_{s}\right)\right) \cap\left(U \cap V_{2}\right)=\emptyset$. Therefore, we can improve (4) as follows:

$$
d_{C}\left(x_{l}\right)+d_{C}\left(x_{s}\right) \leqslant|C|-\sum_{i \in I \backslash\{l, s\}}\left|D_{i}\right|-\left|U \cap V_{2}\right| .
$$

By (2),

$$
\sum_{i \in I \backslash\{l, s\}} d_{C}\left(x_{i}\right) \leqslant \sum_{i \in I \backslash\{l, s\}}\left|D_{i}\right|+(k-2)(\alpha(G)-1) .
$$

By the definition of $x_{i}$, we clearly have $N_{H_{0}}\left(x_{i}\right)=\emptyset$ for $i \in I$. Hence we improve (3) as follows:

$$
\sum_{i \in I} d_{H}\left(x_{i}\right) \leqslant|H|-\left|H_{0}\right|
$$

Hence, by the above four inequalities, we deduce $\sum_{i \in I} d_{G}\left(x_{i}\right)+d_{G}\left(y_{0}\right) \leqslant n+\kappa(G)+(k-$ $2)(\alpha(G)-1)-1$, a contradiction. 
By Claim 12,

there exists an integer $r$ such that $C\left(x_{r}, u_{r}^{\prime}\right] \cap V_{2} \neq \emptyset$,

say

$$
v_{2} \in C\left(x_{r}, u_{r}^{\prime}\right] \cap V_{2} .
$$

Choose $r$ and $v_{2}$ so that $v_{2} \neq u_{r}^{\prime}$ if possible. Note that

$$
d_{G}\left(v_{2}\right) \leqslant\left|V_{2} \cup S\right|-1 \text {. }
$$

By Lemma 7 and Claim 12, we can improve (3) as follows:

$$
\sum_{0 \leqslant i \leqslant m} d_{H}\left(x_{i}\right)+\sum_{w \in W} d_{H}(w) \leqslant|H|-\left|\left\{x_{0}\right\}\right|=\left|H \cap\left(V_{1} \cup S\right)\right|-1 .
$$

By Claim 12 and (1), we can improve (7) as follows:

$$
\begin{aligned}
d_{C}\left(x_{l}\right) & \leqslant\left|C \cap\left(V_{1} \cup S\right)\right|-\sum_{i \in[m] \backslash\{l\}}\left|D_{i}\right|-|X| \\
& \leqslant\left|C \cap\left(V_{1} \cup S\right)\right|-\sum_{i \in[m] \backslash\{l\}}\left|D_{i}\right|-d_{C}\left(x_{0}\right) .
\end{aligned}
$$

Claim 13. $d_{C}\left(x_{0}\right)=|U|=|X|=\alpha(G)-1$. In particular, $N_{C}\left(x_{0}\right)=U$.

Proof. We first show that $d_{C}(w) \leqslant d_{C}\left(x_{0}\right)$ for each $w \in W$. Let $w \in W$. Without loss of generality, we may assume that $w \in W_{1}$. Then by applying Lemma 5 as $Q_{1}:=D_{1}$, $Q_{2}:=D_{2}$ and

$$
D:=x_{1} C\left[w^{+}, u_{2}\right] P\left[u_{2}, u_{1}\right] \overleftarrow{C}\left[u_{1}, x_{2}\right] \overleftarrow{C}\left[w^{-}, x_{1}\right]
$$

where $P\left[u_{2}, u_{1}\right]$ is a $C$-path passing through a vertex of $H_{0}$, we can obtain a cycle $C^{\prime}$ such that $V(C) \backslash\{w\} \subseteq V\left(C^{\prime}\right)$ and $V\left(C^{\prime}\right) \cap V\left(H_{0}\right) \neq \emptyset$ (note that (I) and (II) of Lemma 5 hold, by Lemma 7 (i) and (ii)). Note that $\left|C^{\prime}\right|=|C|$ by the maximality of $|C|$. Note also that $d_{C^{\prime}}(w) \geqslant d_{C}(w)$. By the choice of $C$ and $x_{0}$, we have $d_{C^{\prime}}(w) \leqslant d_{C}\left(x_{0}\right)$, and hence $d_{C}(w) \leqslant d_{C}\left(x_{0}\right)$.

We next show that $d_{C}\left(x_{0}\right)=|U|=|X|=\alpha(G)-1$. By (1), it suffices to prove that $d_{C}\left(x_{0}\right) \geqslant \alpha(G)-1$. Suppose that $d_{C}\left(x_{0}\right) \leqslant \alpha(G)-2$. In this proof, we assume $x_{l}=x_{1}$.

We divide the proof into two cases.

Case 1. $|W| \geqslant \kappa(G)+k-4$.

By the assumption of Case 1 and by Claim 12, we obtain

$$
\begin{aligned}
\left|\left(W \cup\left\{x_{0}, x_{1}, x_{2}, x_{3}\right\}\right) \cap V_{1}\right| & =\left|W \cup\left\{x_{0}, x_{1}, x_{2}, x_{3}\right\}\right|-\left|\left(W \cup\left\{x_{0}, x_{1}, x_{2}, x_{3}\right\}\right) \cap S\right| \\
& \geqslant(\kappa(G)+k-4+4)-\kappa(G)=k .
\end{aligned}
$$


Let $W^{\prime}$ be a $k$-subset of $\left(W \cup\left\{x_{0}, x_{1}, x_{2}, x_{3}\right\}\right) \cap V_{1}$ such that $x_{1} \in W^{\prime}$. Since $W^{\prime} \subseteq V_{1}$ and $v_{2} \in V_{2}$, and by Claim $9, W^{\prime} \cup\left\{v_{2}\right\}$ is independent. Since $d_{C}(w) \leqslant d_{C}\left(x_{0}\right)$ for each $w \in W$, it follows from (10) that

$$
d_{C}\left(x_{1}\right) \leqslant\left|C \cap\left(V_{1} \cup S\right)\right|-\sum_{i \in\{2,3\}}\left|D_{i}\right|-d_{C}\left(w_{0}\right),
$$

where $w_{0} \in W^{\prime} \backslash\left\{x_{1}, x_{2}, x_{3}\right\}$ (note that $\left|W^{\prime}\right|=k \geqslant 4$ ). By (1) and (2),

$$
\begin{aligned}
\sum_{x \in W^{\prime} \backslash\left\{x_{1}, w_{0}\right\}} d_{C}(x) & =\sum_{x \in W^{\prime} \cap\left\{x_{2}, x_{3}\right\}} d_{C}(x)+\sum_{w \in W^{\prime} \backslash\left\{w_{0}, x_{1}, x_{2}, x_{3}\right\}} d_{C}(w) \\
& \leqslant \sum_{i \in\{2,3\}}\left|D_{i}\right|+(k-2)(\alpha(G)-1) .
\end{aligned}
$$

By the above two inequalities, we obtain

$$
\sum_{w \in W^{\prime}} d_{C}(w) \leqslant\left|C \cap\left(V_{1} \cup S\right)\right|+(k-2)(\alpha(G)-1) .
$$

Therefore, since $\sum_{w \in W^{\prime}} d_{H}(w) \leqslant\left|H \cap\left(V_{1} \cup S\right)\right|-1$ by (9), it follows that

$$
\sum_{w \in W^{\prime}} d_{G}(w) \leqslant\left|V_{1} \cup S\right|+(k-2)(\alpha(G)-1)-1 .
$$

Summing this inequality and (8) yields that $\sum_{w \in W^{\prime}} d_{G}(w)+d_{G}\left(v_{2}\right) \leqslant n+\kappa(G)+(k-$ $2)(\alpha(G)-1)-2$, a contradiction.

Case 2. $|W| \leqslant \kappa(G)+k-5$.

By Claim 10, we can take a $(k-3)$-subset $Z$ of $W \cup L$ so that $|W \cap Z|$ is as large as possible. Let $W^{*}:=Z \cap W, L^{*}:=Z \cap L$ and $I^{*}:=\left\{i: x_{i} \in L^{*}\right\}$. By Claim 9, $Z \cup\left\{x_{0}, x_{1}, x_{2}, x_{3}\right\}$ is independent. By (6), we have

$$
d_{C}\left(x_{1}\right)+d_{C}\left(x_{2}\right)+d_{C}\left(x_{3}\right) \leqslant|C|+|W|+\left|L^{*}\right|-\sum_{i \in I^{*}}\left|D_{i}\right|+3 .
$$

On the other hand, since $d_{C}(w) \leqslant d_{C}\left(x_{0}\right)$ for $w \in W$ and $d_{C}\left(x_{0}\right) \leqslant \alpha(G)-2$, it follows from (2) that

$$
\begin{aligned}
\sum_{w \in W^{*} \cup\left\{x_{0}\right\}} d_{C}(w)+\sum_{i \in I^{*}} d_{C}\left(x_{i}\right) & \leqslant\left(\left|W^{*}\right|+1\right)(\alpha(G)-2)+\sum_{i \in I^{*}}\left|D_{i}\right|+\left|L^{*}\right|(\alpha(G)-1) \\
& =(k-2)(\alpha(G)-1)-\left|W^{*}\right|-1+\sum_{i \in I^{*}}\left|D_{i}\right| .
\end{aligned}
$$

Thus, we deduce

$$
\sum_{v \in Z \cup\left\{x_{0}, x_{1}, x_{2}, x_{3}\right\}} d_{C}(v) \leqslant|C|+|W|+\left|L^{*}\right|-\left|W^{*}\right|+(k-2)(\alpha(G)-1)+2 .
$$


If $W \subseteq Z$, then $W=W^{*}$ and $\left|L^{*}\right| \leqslant|Z|=k-3$, and hence

$$
\begin{aligned}
\sum_{v \in Z \cup\left\{x_{0}, x_{1}, x_{2}, x_{3}\right\}} d_{C}(v) & \leqslant|C|+\left|L^{*}\right|+(k-2)(\alpha(G)-1)+2 \\
& \leqslant|C|+(k-3)+(k-2)(\alpha(G)-1)+2 \\
& \leqslant|C|+\kappa(G)+(k-2)(\alpha(G)-1)-1 ;
\end{aligned}
$$

otherwise, $L^{*}=\emptyset$ and $\left|W^{*}\right|=|Z|=k-3$, and so by the assumption of Case 2 ,

$$
\begin{aligned}
\sum_{v \in Z \cup\left\{x_{0}, x_{1}, x_{2}, x_{3}\right\}} d_{C}(v) & \leqslant|C|+|W|-\left|W^{*}\right|+(k-2)(\alpha(G)-1)+2 \\
& \leqslant|C|+\kappa(G)+k-5-(k-3)+(k-2)(\alpha(G)-1)+2 \\
& =|C|+\kappa(G)+(k-2)(\alpha(G)-1) .
\end{aligned}
$$

Therefore, by (9), we have $\sum_{v \in Z \cup\left\{x_{0}, x_{1}, x_{2}, x_{3}\right\}} d_{G}(v) \leqslant n+\kappa(G)+(k-2)(\alpha(G)-1)-1$, a contradiction.

Claim 14. $W \subseteq X$.

Proof. If $W \backslash X \neq \emptyset$, then by Claim 9, we have $|X| \leqslant \alpha(G)-2$, which contradicts Claim 13.

Note that $W^{-} \subseteq U$ by Claim 14 and Lemma 7 (i) (see Figure 4 ).

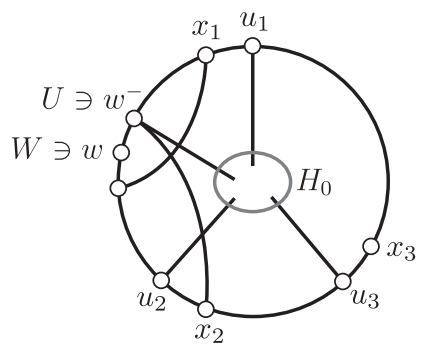

Figure 4: $W^{-} \subseteq U$.

Claim 15. If $u_{s} \in N_{C}\left(x_{t}\right)$ for some $s, t \in[m]$, then $N_{C}\left(x_{s}\right) \cap C\left[u_{t}, u_{s}\right] \subseteq U$.

Proof. Suppose that there exists a vertex $z \in N_{C}\left(x_{s}\right) \cap C\left[u_{t}, u_{s}\right]$ such that $z \notin U$ (see Figure 5 (i)). Since $z \notin U$, it follows from Lemma 7 (i) that $z^{+} \notin X$. By Claim 13, $X \cup\left\{x_{0}, z^{+}\right\}$is not an independent set. Hence $z^{+} \in N_{C}\left(x_{h}\right)$ for some $x_{h} \in X \cup\left\{x_{0}\right\}$. Since $x_{s}$ is a non-insertible vertex, it follows that $x_{h} \neq x_{s}$. Let $z_{s}$ be the vertex in $C\left(u_{s}, x_{s}\right]$ such that $z \in N_{G}\left(z_{s}\right)$ and $z \notin N_{G}(v)$ for all $v \in C\left(u_{s}, z_{s}\right)$. By Lemma 7 (ii), we obtain $x_{h} \notin C\left[u_{s}^{\prime}, z\right]$. Therefore, $x_{h} \in C\left(z, u_{s}\right] \cup\left\{x_{0}\right\}$. If $x_{h} \in C\left(z, u_{s}\right]$, then we let $z_{h}$ be the 
vertex in $C\left(u_{h}, x_{h}\right]$ such that $z^{+} \in N_{G}\left(z_{h}\right)$ and $z^{+} \notin N_{G}(v)$ for all $v \in C\left(u_{h}, z_{h}\right)$. We define the cycle $C^{*}$ as follows (see Figure 5 (ii) and (iii)):

$$
C^{*}= \begin{cases}z_{s} \overleftarrow{C}\left[z, x_{t}\right] \overleftarrow{C}\left[u_{s}, z_{h}\right] C\left[z^{+}, u_{h}\right] x_{0} \overleftarrow{C}\left[u_{t}, z_{s}\right] & \text { if } x_{h} \in C\left(z, u_{s}\right] \\ z_{s} \overleftarrow{C}\left[z, x_{t}\right] \overleftarrow{C}\left[u_{s}, z^{+}\right] x_{h} \overleftarrow{C}\left[u_{t}, z_{s}\right] & \text { if } x_{h}=x_{0}\end{cases}
$$

Then, by a similar argument in the proof of Lemma 7, we can obtain a longer cycle than $C$ by inserting all vertices of $V\left(C \backslash C^{*}\right)$ into $C^{*}$. This contradicts that $C$ is longest.

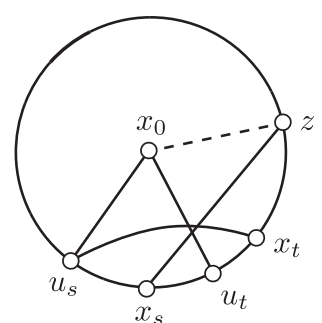

(i)

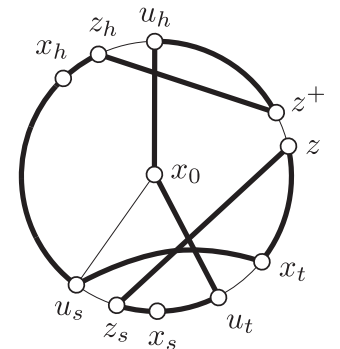

(ii)

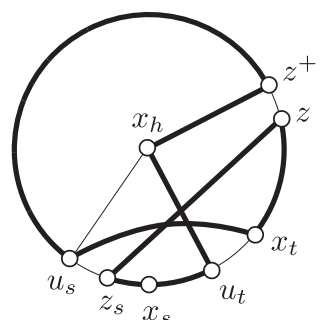

(iii)

Figure 5: (i) A vertex $z \in N_{C}\left(x_{s}\right) \cap C\left[u_{t}, u_{s}\right]$ such that $z \notin U$, (ii) the cycle $C^{*}$ in the case $x_{h} \in C\left(z, u_{s}\right]$ and (iii) the case $x_{h}=x_{0}$.

Notice that for each vertex $x_{i} \in W \cup L$, there exists $j \in[m] \backslash\{i\}$ such that $u_{i} \in N_{C}\left(x_{j}\right)$, and hence Claim 15 implies that $N_{C}\left(x_{i}\right) \cap C\left[u_{j}, u_{i}\right] \subseteq U$.

We divide the rest of the proof into two cases.

Case 1. $v_{2} \notin U$.

Let $Y:=N_{G}\left(v_{2}\right) \cap X$, and let $\gamma:=|X|-\kappa(G)-1$. Note that $x_{l} \notin Y$ since $x_{l} \in V_{1}$.

Claim 16. $|Y| \geqslant \gamma+3$.

Proof. Suppose that $|Y| \leqslant \gamma+2$. By the assumption of Case 1 and Claim 13, we have $x_{0} v_{2} \notin E(G)$. Since $\left|X \cup\left\{x_{0}\right\}\right| \geqslant k+\gamma+2$ and $|Y| \leqslant \gamma+2$, there exists a set $I$ of $k$ integers such that $\left\{x_{0}, x_{l}\right\} \subseteq\left\{x_{i}: i \in I\right\} \subseteq\left(X \cup\left\{x_{0}\right\}\right) \backslash Y$. Then $\left\{x_{i}: i \in I\right\} \cup\left\{v_{2}\right\}$ is independent. Therefore, it follows from (2) and (10) that

$$
\sum_{i \in I} d_{C}\left(x_{i}\right) \leqslant\left|C \cap\left(V_{1} \cup S\right)\right|+(k-2)(\alpha(G)-1) .
$$

Hence, by this inequality and (8) and (9), we obtain

$$
\sum_{i \in I} d_{G}\left(x_{i}\right)+d_{G}\left(v_{2}\right) \leqslant n+\kappa(G)+(k-2)(\alpha(G)-1)-2,
$$

a contradiction. 
In the rest of Case 1 , we assume that $l=1$. If $u_{r}^{\prime} \neq u_{1}$, then let $r=2$ and $u_{3}=u_{2}^{\prime}$; otherwise, let $r=3$ and let $u_{2}$ be the vertex with $u_{2}^{\prime}=u_{3}$. By Claim 14, we obtain $Y \cup W \cup L \subseteq X \backslash\left\{x_{1}\right\}$. Therefore, by Claims 10 and 16 and by the definition of $\gamma$, we obtain

$$
\begin{aligned}
|Y \cap(W \cup L)| & =|Y|+|W \cup L|-|Y \cup(W \cup L)| \\
& \geqslant \gamma+3+\kappa(G)-2-\left|X \backslash\left\{x_{1}\right\}\right| \\
& =\gamma+3+\kappa(G)-2-((\kappa(G)+\gamma+1)-1)=1 .
\end{aligned}
$$

Hence there exists a vertex $x_{h} \in Y \cap(W \cup L)$, that is, $v_{2} \in N_{C}\left(x_{h}\right) \backslash U$. Note that if $x_{h} \in L$ then by the definition of $L, u_{h} \in N_{C}\left(\left\{x_{1}, x_{2}, x_{3}\right\}\right)$; if $x_{h} \in W$ then by the definition of $W$ and Claim 14, $x_{h}^{-}=u_{h} \in N_{C}\left(\left\{x_{1}, x_{2}, x_{3}\right\}\right)$ (see Figure 4 and the paragraph below Claim 15). Since $C\left(x_{2}, x_{3}\right) \cap X=\emptyset$ and $C\left(x_{3}, x_{1}\right) \cap X=\emptyset$ if $r=3$, either $u_{h} \in N_{C}\left(x_{1}\right)$ and $u_{h} \in C\left(x_{3}, u_{1}\right)$ or $u_{h} \in N_{C}\left(x_{2}\right)$ and $u_{h} \in C\left(x_{1}, u_{2}\right)$ holds (especially, if $r=3$ then the latter case holds).

If $r=2$ and $u_{h} \in N_{C}\left(x_{1}\right)$, then $v_{2} \in C\left[u_{1}, u_{h}\right]$ (see Figure 6 (i)). If $r=2$ and $u_{h} \in N_{C}\left(x_{2}\right)$, then $v_{2} \in C\left[u_{2}, u_{h}\right]$ (see Figure 6 (ii)). If $r=3$, then $u_{h} \in N_{C}\left(x_{2}\right)$ and $v_{2} \in C\left[u_{2}, u_{h}\right]$ (see Figure 6 (iii)). In each case, we obtain a contradiction to Claim 15.

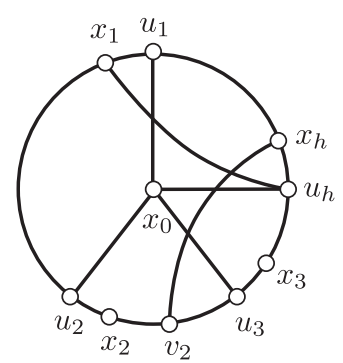

(i)

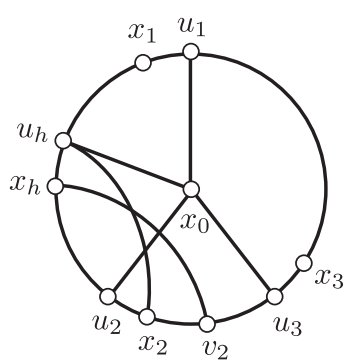

(ii)

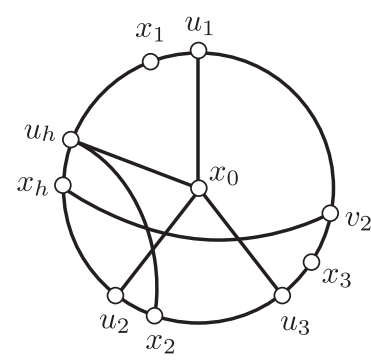

(iii)

Figure 6: (i) The case $r=2$ and $u_{h} \in N_{C}\left(x_{1}\right)$, (ii) the case $r=2$ and $u_{h} \in N_{C}\left(x_{2}\right)$, and (iii) the case $r=3$.

Case 2. $v_{2} \in U$.

We first show that $N_{C}\left(x_{i}\right) \cap\left(U \backslash\left\{u_{i}\right\}\right) \neq \emptyset$ for each $x_{i} \in X$. For $x_{i} \in X$, let $x_{i}^{\prime}$ and $x_{i}^{\prime \prime}$ be the successors of $x_{i}$ and $x_{i}^{\prime}$ in $X$ along the orientation of $C$, respectively. Let $x_{1}=x_{i}$, $x_{2}=x_{i}^{\prime}$ and $x_{3}=x_{i}^{\prime \prime}$. Then by Claim 10, it follows that $W \cup L \neq \emptyset$. By the definition of $x_{i}^{\prime}$ and $x_{i}^{\prime \prime}$, and Claim 14, we have $W_{1}=W_{2}=\emptyset$ (note that $W \cap\left\{x_{1}, x_{2}, x_{3}\right\}=\emptyset$ ). By the definitions of $x_{i}^{\prime}, x_{i}^{\prime \prime}, L_{1}$ and $L_{2}$, we also have $L_{1}=L_{2}=\emptyset$. Thus $W_{3} \cup L_{3} \neq \emptyset$. By Lemma 7 (i) and since $W \cup L \subseteq X$, this implies that $N_{C}\left(x_{i}\right) \cap\left(U \backslash\left\{u_{i}\right\}\right) \neq \emptyset$.

We rename $x_{i} \in X$ for $i \geqslant 1$ as follows (see Figure 7 (i)): Rename an arbitrary vertex of $X$ as $x_{1}$ (but we will re-choose $x_{1}$ later). For $i \geqslant 1$, we rename $x_{i+1} \in X$ so that $u_{i+1} \in N_{C}\left(x_{i}\right) \cap\left(U \backslash\left\{u_{i}\right\}\right)$ and $\left|C\left[u_{i+1}, x_{i}\right)\right|$ is as small as possible. Let $h$ be the minimum integer such that $x_{h+1} \in C\left(x_{h}, x_{1}\right]$. Note that this choice implies $h \geqslant 2$. We rename $h$ 
vertices in $X$ as $\left\{x_{1}, x_{2}, \ldots, x_{h}\right\}$ as above (Note that the order is in opposite direction of $C$.), and $m-h$ vertices in $X \backslash\left\{x_{1}, x_{2}, \ldots, x_{h}\right\}$ as $\left\{x_{h+1}, x_{h+2}, \ldots, x_{m}\right\}$ arbitrarily. Set

$$
A_{1}:=A_{h+1}:=C\left[x_{1}, x_{h}\right) \quad \text { and } \quad A_{i}:=C\left[x_{i}, x_{i-1}\right) \quad \text { for } 2 \leqslant i \leqslant h
$$

(see Figure 7 (ii)).

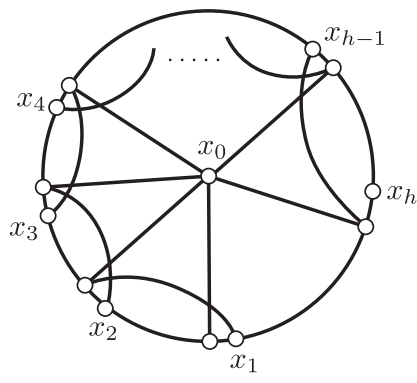

(i)

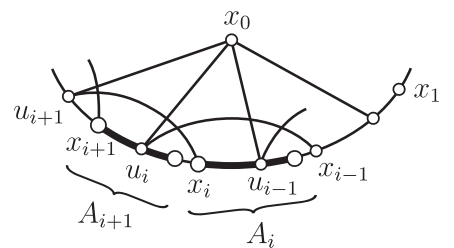

(ii)

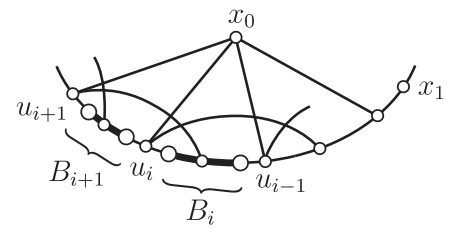

(iii)

Figure 7: The definition: (i) $x_{1}, x_{2}, \ldots, x_{h}$, (ii) $A_{i}$, and (iii) $B_{i}$.

We divide the proof of Case 2 according to whether $h \leqslant k$ or $h \geqslant k+1$.

Subcase 2.1. $h \leqslant k$.

By the definition of $\left\{x_{1}, \ldots, x_{h}\right\}$, we have

$$
N_{A_{i+1}}\left(x_{i}\right) \cap U \subseteq\left\{u_{i}\right\} \quad \text { for } 1 \leqslant i \leqslant h .
$$

By Claim 15 and (11), we obtain

$$
N_{C \backslash A_{i}}\left(x_{i}\right) \subseteq\left(U \backslash\left(A_{i} \cup A_{i+1}\right)\right) \cup D_{i} \cup\left\{u_{i}\right\} \text { for } 2 \leqslant i \leqslant h .
$$

By Lemma 7 (i) and (ii), $N_{A_{i}}\left(x_{i}\right)^{-} \cap N_{A_{i}}\left(x_{1}\right)=\emptyset$ for $2 \leqslant i \leqslant h$. By Lemma 7 (i), we have $N_{A_{i}}\left(x_{i}\right)^{-} \cup N_{A_{i}}\left(x_{1}\right) \subseteq A_{i} \backslash D$ for $3 \leqslant i \leqslant h$. Thus, it follows from (12) that for $3 \leqslant i \leqslant h$

$$
d_{C}\left(x_{i}\right) \leqslant\left(|U|-\left|\left(A_{i} \cup A_{i+1}\right) \cap U\right|+\left|D_{i}\right|+1\right)+\left(\left|A_{i}\right|-\left|A_{i} \cap D\right|-d_{A_{i}}\left(x_{1}\right)\right) .
$$

By Lemma 7 (i) and (11), we have $N_{A_{2}}\left(x_{2}\right)^{-} \cup N_{A_{2}}\left(x_{1}\right) \subseteq\left(A_{2} \backslash(U \cup D)\right) \cup D_{1} \cup\left\{u_{1}\right\}$. Thus, by (12), we have

$$
\begin{aligned}
d_{C}\left(x_{2}\right) \leqslant & \left(|U|-\left|\left(A_{2} \cup A_{3}\right) \cap U\right|+\left|D_{2}\right|+1\right) \\
& +\left(\left|A_{2}\right|-\left|A_{2} \cap U\right|-\left|A_{2} \cap D\right|+\left|D_{1}\right|+1-d_{A_{2}}\left(x_{1}\right)\right) .
\end{aligned}
$$

Since $\left|A_{1} \cap X\right|=\left|A_{1} \cap U\right|$, it follows from Lemma 7 (i) that

$$
d_{A_{1}}\left(x_{1}\right) \leqslant\left|A_{1}\right|-\left|A_{1} \cap D\right|-\left|A_{1} \cap X\right|=\left|A_{1}\right|-\left|A_{1} \cap D\right|-\left|A_{1} \cap U\right| .
$$


By Claim 13, $d_{C}\left(x_{0}\right)=|U|=\alpha(G)-1$. Thus, since $h \leqslant k$, we obtain

$$
\begin{aligned}
\sum_{0 \leqslant i \leqslant h} d_{C}\left(x_{i}\right) & \leqslant \sum_{1 \leqslant i \leqslant h}\left|A_{i}\right|+h|U|-2 \sum_{1 \leqslant i \leqslant h}\left|A_{i} \cap U\right|+h+\sum_{1 \leqslant i \leqslant h}\left|D_{i}\right|-\sum_{1 \leqslant i \leqslant h}\left|A_{i} \cap D\right| \\
& =|C|+(h-2)|U|+h+\sum_{1 \leqslant i \leqslant h}\left|D_{i}\right|-|D| \\
& \leqslant|C|+k+(h-2)(\alpha(G)-1)+\sum_{1 \leqslant i \leqslant h}\left|D_{i}\right|-|D| .
\end{aligned}
$$

Let $I$ be a $(k+1)$-subset of $\{0,1, \ldots, m\}$ such that $\{0,1, \ldots, h\} \subseteq I$. By Claim 8 , $\left\{x_{i}: i \in I\right\}$ is independent. By the above inequality and (2), we have

$$
\sum_{i \in I} d_{C}\left(x_{i}\right) \leqslant|C|+k+(k-2)(\alpha(G)-1)
$$

Hence, by (3), we obtain $\sum_{i \in I} d_{G}\left(x_{i}\right) \leqslant|G|+\kappa(G)+(k-2)(\alpha(G)-1)-1$, a contradiction.

Subcase 2.2. $h \geqslant k+1$.

We first set

$$
U_{1}:=\left\{u_{i} \in U: x_{i} \in X \cap V_{1}\right\} .
$$

Choose $x_{1}$ so that $A_{2} \cap U_{1}=\emptyset$ if possible.

By the assumption of Case 2 and the choice of $r$ and $v_{2}$ (see the paragraph below the proof of Claim 12), we have $V_{2} \cap \bigcup_{i=1}^{m} C\left(x_{i}, u_{i}^{\prime}\right)=\emptyset$. Hence, it follows from Claims 12 and 13 that $V_{2} \subseteq N_{C}\left(x_{0}\right)$. Since $x_{0} \in V_{1} \cup S$ by Claim 12, this implies that $x_{0} \in S$.

Claim 17. $\left|X \cap V_{1}\right| \leqslant k-1$.

Proof. Suppose that $\left|X \cap V_{1}\right| \geqslant k$. Let $I$ be a $k$-subset of $[m]$ such that $I \subseteq\left\{i: x_{i} \in\right.$ $\left.X \cap V_{1}\right\}$. Then $\left\{x_{i}: i \in I\right\} \cup\left\{v_{2}\right\}$ is independent. Let $s$ and $t$ be distinct integers in $I$. Since $\left\{x_{s}, x_{t}\right\} \subseteq V_{1}$ and $D \subseteq V_{1} \cup S$, the similar argument as that of (4) implies that

$$
d_{C}\left(x_{s}\right)+d_{C}\left(x_{t}\right) \leqslant\left|C \cap\left(V_{1} \cup S\right)\right|-\sum_{i \in I \backslash\{s, t\}}\left|D_{i}\right| .
$$

By (2) and (9), we have $\sum_{i \in I \backslash\{s, t\}} d_{C}\left(x_{i}\right) \leqslant \sum_{i \in I \backslash\{s, t\}}\left|D_{i}\right|+(k-2)(\alpha(G)-1)$ and $\sum_{i \in I} d_{H}\left(x_{i}\right) \leqslant\left|H \cap\left(V_{1} \cup S\right)\right|-1$, respectively. On the other hand, we obtain $d_{G}\left(v_{2}\right) \leqslant \mid V_{2} \cup$ $S \mid-1$. By these four inequalities, $\sum_{i \in I} d_{G}\left(x_{i}\right)+d_{G}\left(v_{2}\right) \leqslant n+\kappa(G)+(k-2)(\alpha(G)-1)-2$, a contradiction. Therefore $\left|X \cap V_{1}\right| \leqslant k-1$.

By Claim 17, we have $\left|U_{1}\right| \leqslant k-1$. Therefore, by the assumption of Subcase 2.2 and the choice of $x_{1}$, we obtain $A_{2} \cap U_{1}=\emptyset$, and hence we can take a $k$-subset $I$ of $\{2,3, \ldots, h\}$ such that $\left\{i: A_{i+1} \cap U_{1} \neq \emptyset\right\} \subseteq I$. Let

$$
X_{I}:=\left\{x_{i}: i \in I\right\}
$$


By Claim 8, $X_{I} \cup\left\{x_{0}\right\}$ is independent. Set

$$
B_{1}:=B_{h+1}:=C\left(u_{1}, u_{h}\right) \quad \text { and } \quad B_{i}:=C\left(u_{i}, u_{i-1}\right) \quad \text { for } 2 \leqslant i \leqslant h
$$

(see Figure 7 (iii)). Then, since $\left|C\left[u_{i}, u_{i}^{\prime}\right)\right| \geqslant 2$ for $i \in[m] \backslash I$, the following inequality holds:

$$
\begin{aligned}
|C| & \geqslant \sum_{i \in I}\left|B_{i} \cup\left\{u_{i}\right\}\right|+2\left(|U|-\sum_{i \in I}\left|\left(B_{i} \cup\left\{u_{i}\right\}\right) \cap U\right|\right) \\
& =\sum_{i \in I}\left|B_{i}\right|+2\left(|U|-\sum_{i \in I}\left|B_{i} \cap U\right|\right)-k .
\end{aligned}
$$

By the definition of $\left\{x_{1}, \ldots, x_{h}\right\}$, we have $N_{B_{i+1}}\left(x_{i}\right) \cap U=\emptyset$ for $1 \leqslant i \leqslant h$. If $x_{i} \in X_{I} \cap S$, then it follows from Lemma 7 (i) and Claim 15 that

$$
\begin{aligned}
d_{C}\left(x_{i}\right) & \leqslant\left(|U|-\left|B_{i} \cap U\right|-\left|B_{i+1} \cap U\right|\right)+\left(\left|B_{i}\right|-\left|\left\{x_{i}\right\}\right|-\left|\left(B_{i} \cap U\right)^{+}\right|\right) \\
& =|U|+\left|B_{i}\right|-2\left|B_{i} \cap U\right|-\left|B_{i+1} \cap U\right|-1 .
\end{aligned}
$$

If $x_{i} \in X_{I} \cap V_{1}$, then by Lemma 7 (i) and Claim 15,

$$
\begin{aligned}
d_{C}\left(x_{i}\right) \leqslant & \left(|U|-\left|B_{i} \cap U\right|-\left|B_{i+1} \cap U\right|-\left|\left(U \cap V_{2}\right) \backslash\left(B_{i} \cup B_{i+1}\right)\right|\right) \\
& +\left(\left|B_{i}\right|-\left|\left\{x_{i}\right\}\right|-\left|\left(B_{i} \cap U\right)^{+}\right|-\left|\left(U \cap V_{2}\right) \cap B_{i}\right|\right) \\
= & |U|+\left|B_{i}\right|-2\left|B_{i} \cap U\right|-\left|B_{i+1} \cap U\right|-1-\left|\left(U \cap V_{2}\right) \backslash B_{i+1}\right| .
\end{aligned}
$$

Since $U \cap V_{2} \neq \emptyset$, we obtain $\left|\left(U \cap V_{2}\right) \backslash B_{i+1}\right| \geqslant 1$ for all $i \in I$ except for at most one, and hence

$$
\sum_{i \in I: x_{i} \in X_{I} \cap V_{1}}\left|\left(U \cap V_{2}\right) \backslash B_{i+1}\right| \geqslant\left|X_{I} \cap V_{1}\right|-1
$$

By the choice of $I$, we have

$$
\left|U_{1}\right|=\sum_{i \in I}\left|A_{i+1} \cap U_{1}\right|=\sum_{i \in I}\left|B_{i+1} \cap U_{1}\right|+\left|X_{I} \cap V_{1}\right| \leqslant \sum_{i \in I}\left|B_{i+1} \cap U\right|+\left|X_{I} \cap V_{1}\right| .
$$

On the other hand, since $x_{0} \in S$, it follows from Claim 12 that

$$
\left|U_{1}\right|=\left|X \cap V_{1}\right|=|X \backslash S| \geqslant|X|-(\kappa(G)-1),
$$

and hance

$$
\sum_{i \in I}\left|B_{i+1} \cap U\right|+\left|X_{I} \cap V_{1}\right| \geqslant\left|U_{1}\right| \geqslant|X|-(\kappa(G)-1) .
$$

Moreover, by Claim 13, $d_{C}\left(x_{0}\right)=|U|=|X|=\alpha(G)-1$. Thus, we deduce

$$
\sum_{i \in I \cup\{0\}} d_{C}\left(x_{i}\right) \leqslant(k+1)|U|+\sum_{i \in I}\left|B_{i}\right|-2 \sum_{i \in I}\left|B_{i} \cap U\right|
$$




$$
\begin{aligned}
& -\sum_{i \in I}\left|B_{i+1} \cap U\right|-k-\left(\left|X_{I} \cap V_{1}\right|-1\right) \\
= & \left(\sum_{i \in I}\left|B_{i}\right|+2\left(|U|-\sum_{i \in I}\left|B_{i} \cap U\right|\right)-k\right)+(k-1)|U| \\
& -\left(\sum_{i \in I}\left|B_{i+1} \cap U\right|+\left|X_{I} \cap V_{1}\right|\right)+1
\end{aligned}
$$

and hence, by (13) and (14),

$$
\begin{aligned}
\sum_{i \in I \cup\{0\}} d_{C}\left(x_{i}\right) & \leqslant|C|+(k-1)|U|-(|X|-\kappa(G)+1)+1 \\
& =|C|+\kappa(G)+(k-2)(\alpha(G)-1) .
\end{aligned}
$$

Hence, by (3), we obtain $\sum_{i \in I \cup\{0\}} d_{G}\left(x_{i}\right) \leqslant|G|+\kappa(G)+(k-2)(\alpha(G)-1)-1$, a contradiction.

\section{Acknowledgements}

The authors would like to thank the referees for valuable suggestions and comments.

\section{References}

[1] A. Ainouche, An improvement of Fraisse's sufficient condition for hamiltonian graphs, J. Graph Theory 16 (1992), 529-543.

[2] D. Bauer, H.J. Broersma, H.J. Veldman and R. Li, A generalization of a result of Häggkvist and Nicoghossian, J. Combin. Theory Ser. B 47 (1989), 237-243.

[3] J.A. Bondy, A remark on two sufficient conditions for Hamilton cycles, Discrete Math. 22 (1978), 191-193.

[4] J.A. Bondy, Longest paths and cycles in graphs with high degree, Research Report CORR 80-16, Department of Combinatorics and Optimization, University of Waterloo, Waterloo, Ontario, Canada (1980).

[5] J.A. Bondy and U.S.R. Murty, Graph Theory, Springer, Berlin, 2008.

[6] V. Chvátal and P. Erdős, A note on hamiltonian circuits, Discrete Math. 2 (1972), 111-113.

[7] G.A. Dirac, Some theorems on abstract graphs, Proc. London Math. Soc. 2 (1952), 69-81.

[8] P. Fraisse and H. A. Jung, "Longest cycles and independent sets in $k$-connected graphs," Recent Studies in Graph Theory, V.R. Kulli (Editor), Vischwa Internat. Publ. Gulbarga, India, 1989, pp. 114-139.

[9] H. Li, Generalizations of Dirac's theorem in Hamiltonian graph theory-A survey, Discrete Math. 313 (2013), 2034-2053. 
[10] O. Ore, Note on Hamilton circuits, Amer. Math. Monthly 67 (1960), 55.

[11] K. Ota, Cycles through prescribed vertices with large degree sum, Discrete Math. 145 (1995), 201-210.

[12] K. Ozeki and T. Yamashita, A degree sum condition concerning the connectivity and the independence number of a graph, Graphs Combin. 24 (2008), 469-483. 